\title{
EXTRACT 2.0: text-mining-assisted interactive annotation of bio- medical named entities and ontology terms
}

\author{
Evangelos Pafilis', ${ }^{1,}$ Rūdolfs Bērzinšš , and Lars Juhl Jensen ${ }^{2, *}$ \\ 1 Institute of Marine Biology, Biotechnology and Aquaculture, Hellenic Centre for Marine Research, P.O. Box \\ 2214, 71003 Heraklion, Crete, Greece \\ ${ }^{2}$ Cellular Network Biology Group, Novo Nordisk Foundation Center for Protein Research, Faculty of Health and \\ Medical Sciences, University of Copenhagen, 2200 Copenhagen N, Denmark
}

\section{INTRODUCTION}

Databases increasingly rely on text-mining tools to support the curation process. The BioCreative interactive annotation task recently evaluated several such tools and found our tool EXTRACT to perform favorably in terms of usability and accelerated curation by $15-25 \%$ (Wang et al., 2016).

The original version of EXTRACT was designed to support annotation of metagenomic samples with semantically controlled environmental descriptors (Pafilis et al., 2016). For this reason, it focused on named entity recognition of terms from the Environment Ontology (ENVO) (Buttigieg et al., 2016) and ontologies relevant for describing host organisms (https://www.ncbi.nlm.nih.gov/taxonomy), tissues (Placzek et al., 2017), and disease states (Kibbe et al., 2015).

\section{EXPANDED SCOPE OF THE TOOL}

EXTRACT 2.0 expands the scope of the tool in several new directions with the aim to make it more broadly useful.

We expanded the scope from covering only diseases to covering phenotypes in general. To this end, we complemented the existing disease dictionary with terms from the Mammalian Phenotype Ontology (MPO) (Smith and Eppig, 2012). To avoid redundancy in the dictionary, we excluded MPO terms that clashed with terms already in the disease dictionary. To improve recall, we added plural and adjective endings to the names and generated variants of the form pronoun of noun from names of the form noun pronoun.

To cover also important concepts of molecular and cellular biology, we further expanded the dictionary with Gene Ontology (GO). The names from GO were processed similar to those from MPO to generate variants and improve recall.

In addition to adding more biomedical ontologies, we have expanded the tool with named entity recognition of molecular entities. To this end, we have included dictionaries of protein-coding and non-coding RNA (ncRNA) genes from STRING (Szkararczyk et al., 2017) and RAIN (Junge et al., 2017), respectively. We have furthermore added a diction-

\footnotetext{
*To whom correspondence should be addressed: pafilis@hcmr.gr, lars.juh1.jensen@cpr.ku.dk
}

ary of drugs and other small molecule compounds from the STITCH database (Szklarczyk et al., 2016).

Together, these additional types of entities have made EXTRACT 2.0 potentially useful for many more tasks than just annotation of metagenomic samples. For example, it can be used to help annotate both proteins and ncRNAs with functions, processes, subcellular localization, tissue expression, and associated diseases. The tool, API, and documentation are freely accessible at http://extract.jensenlab.org/.

\section{FUNDING}

The Novo Nordisk Foundation (NNF14CC0001).

\section{REFERENCES}

Wang,Q., Abdul,S.S., Almeida,L. et al. (2016). Overview of the interactive task in BioCreative V. Database, 2016, baw119.

Pafilis,E., Buttigieg,P.L., Ferrell,B. et al. (2016) EXTRACT: interactive extraction of environment metadata and term suggestion for metagenomic sample annotation. Database, 2016, baw005.

Buttigieg,P.L., Pafilis,E., Lewis,S.E. et al. (2016) The environment ontology in 2016: bridging domains with increased scope, semantic density, and interoperation. J. Biomed. Semant., 7, 57.

Placzek,S., Schomburg,I., Chang,A. et al. (2017) BRENDA in 2017: new perspectives and new tools in BRENDA. Nucleic Acids Res., 45, D380-D388.

Kibbe,W.A., Arze,C., Felix,V. et al. (2015) Disease Ontology 2015 update: an expanded and updated database of human diseases for linking biomedical knowledge through disease data. Nucleic Acids Res., 43, D1071-D1078.

Smith,C.L. and Eppig,J.T. (2012) The Mammalian Phenotype Ontology as a unifying standard for experimental and highthroughput phenotyping data. Mamm. Genome, 23, 654-668.

Szklarczyk,D., Morris,J.H., Cook,H. et al. (2017). The STRING database in 2017: quality-controlled protein-protein association. Nucleic Acids Res., 45, D362-D368.

Junge,A., Refsgaard,J.C., Garde,C. et al. (2017). RAIN: RNAprotein Association and Interaction Networks. Database, 2017, baw167.

Szklarczyk,D., Santos,A., von Mering,C. et al. (2016). STITCH 5: augmenting protein-chemical interaction networks with tissue and affinity data. Nucleic Acids Res., 44, D380-D384. 